\title{
Time-Varying Network Connectedness of G-7 Economic Policy Uncertainties: A Locally Stationary TVP-VAR Approach.
}

\author{
ONUR POLAT
}

Received: 19.10.2021; Revised: 23.11.2021; Accepted: 25.11.2021

\begin{abstract}
This work analyzes the frequency-dependent network structure of Economic Policy Uncertainties (EPU) across G-7 countries between January 1998 and April 2021. We implement an approach that builds dynamic networks relying on a locally stationary Time-Varying Parameter-Vector Autoregressive model using Quasi-Bayesian Local Likelihood methods. We compute short-, medium-, and long-term network connectedness of G-7 EPUs over a period covering several economic/financial turmoils. Furthermore, we structure short-term network topologies for the Global Financial Crisis (GFC) and the COVID-19 pandemic periods. Findings of the study indicate amplified interdependencies between G-7 EPUs around well-known economic/geopolitical incidents, frequency-dependent connectedness networks among them, and stronger interdependencies than the medium-, and long-term linkages. Finally, we find that short-term spillovers are not persistent in the long-term for both turmoil periods.
\end{abstract}

JEL codes: C10, C40, C58

Keywords: Dynamic networks, TVP-VAR, Pairwise spillovers, Financial connectedness

\section{Introduction}

Economic policy uncertainty (EPU) is a vital notion for both emerging and advanced economies particularly in the aftermath of the Global Financial Crisis (GFC). It has gained further prominence thenceforth and kept its essential role in sustaining a healthy and efficient economic system for policymakers and authorities. Monetary, fiscal, and regulatory policy uncertainties have destructive impacts on the economy (Hassett \& Metcalf, 1999; Byrne \& Davis, 2004; Fernández-Villaverde et al., 2015; Balcılar et al., 2017; De Pooter et al., 2021). Additionally, the policy uncertainty has a real effect on the investment and employment decisions of firms and may cause economic disruptions (Bernanke, 1983; Romer, 1990; Abel \& Eberly, 1996). A precursor study in this vein underlines that policy uncertainty can lead to delays in developing countries' investment decisions (Rodrik, 1991).

a This paper is a revised and extended version of a paper entitled "Dynamic Interdependencies Networks of G-7 Economic Policy Uncertainties: Evidence from the TVP-VAR Frequency Connectedness" presented at EconTR2021, Virtual Mode, 2-4 September 2021.

b Department of Public Finance, Bilecik Şeyh Edebali University. email: onur.polat@bilecik.edu.tr (D) https://orcid.org/0000-0002-7170-4254 
The post-GFC era has been accompanied by drastic financial/geopolitical imbalances such as the Eurozone crisis, the recent COVID-19 pandemic, and the EPU has become a core concept to capture an unprecedented financial/geopolitical shock. Financialization and globalization have conduced tightly connected economies via various channels, which have led to rapid risk propagation through them. The tight connectedness among economic agents motivates a strand of recent studies examining the network structure of connectedness during turmoil and burst episodes (Mensi et al., 2018; Kang \& Lee, 2019; Reboredo et al., 2020; Geng et al., 2021). However, such linkages build networks of connections over different horizons and thereby are frequency dependent. Additionally, an unprecedented shock may lead to transitory or more permanent interdependencies between financial agents (Ellington \& Baruník, 2020). Accordingly, such shocks generate frequency-dependent network structures of connectedness among economic agents.

Since an unanticipated shock may create a frequency-dependent network structure between economic agents, which has a potential effect on their preferences, scholars have focused on the network connectedness of financial/geopolitical bursts (Diebold \& Yılmaz, 2014; Demirer et al., 2018; Kang \& Yoon, 2019; Zhang \& Broadstock, 2020; Mensi et al., 2021). Among these financial/geopolitical imbalances, the recent COVID-19 pandemic is arguably the most acute one which has sorely hit the world economy.

The COVID-19 pandemic first emerged in Chinese town Wuhan in late 2019, and the pandemic rapidly spilled to the rest of the world. As of September 8, 2021, the number of confirmed COVID-19 cases has surpassed 221 million with more than 4.5 million deaths. ${ }^{1}$ The pandemic has led to an acute global recession, and the world output is preceded to be around 3\% lower than the pre-pandemic anticipations (Barrett et al., 2021). Owing to the severe contraction of the world economy led by the COVID-19 pandemic, this study aims to investigate the dynamic network structure of EPUs between G-7 countries (Canada, France, Germany, Italy, Japan, the UK, and the US), reflecting two economic upheavals, the GFC and the COVID-19 pandemic. In this context, we employ the network connectedness approach of Ellington \& Baruník (2020), which is based on a locally stationary TimeVarying Parameter-Vector Autoregressive (TVP-VAR) model using Quasi-Bayesian Local Likelihood (QBLL) methods. By doing so, we compute the short-, medium-, and long-term network connectedness of G-7 EPUs over a period covering two financial turmoil epochs. ${ }^{2}$ Additionally, we compare directional spillovers among G-7 countries pertain to G-7 EPU connectedness networks of the GFC and the COVID-19 episodes.

We contribute to the extant literature in three ways. First, we estimate the dynamic network connectedness of G-7 EPUs over different frequencies by employing a seminal approach. This novel methodology allows us to combine prior shrinkage and to draw the posterior distribution of the dynamic adjacency matrix. Second, we identify the dynamic network structure of G-7 EPUs for the GFC and the COVID-19 pandemic eras and compare spillovers in the sense of their magnitude. Third, we concentrate on the dynamic network connectedness of pairwise spillovers changing over time.

The rest of the paper is organized as follows. The next section presents related studies on the network connectedness of financial/economic indicators. Section 3 provides the method-

1 See, https://covid19.who.int/.

2 Short-, medium-, and long-term connectedness roughly corresponds to 1-6 months, 7-12 months, and 12+ months, respectively. 
ology and the data, Section 4 discusses the empirical findings, and section 5 concludes.

\section{Related Literature}

Globalization along with financialization has led to strongly connected financial markets via various channels, which propels expeditious risk propagation during financial imbalance periods. In this context, a strand of literature focuses on financial contagion during turbulence times and detect intensified connectedness between financial indicators (Billio et al., 2012; Cimini, 2015; Singh et al., 2018; Gong et al., 2019; Bagheri \& Ebrahimi, 2020; Balcular et al., 2021).

It should be noted that scholars have utilized the network theory in various fields, such as sociology (Murdoch, 2001; Mützel, 2009; Weiske et al., 2015), technology (Cresswell et al., 2010; Shim \& Shin, 2016; Beaman et al., 2021), health (Valente \& Pitts, 2017; Iyamu \& Mgudlwa, 2018), and education (Fenwick, 2010; Zhang \& Heydon, 2016). The network theory is a relatively new notion for economics and finance fields, and recent studies have implemented the network theory to analyze the connectedness between indicators. In this vein, Kuzubaş et al. (2014) utilized the network theory to examine the Turkish Interbank market during the 2000 Turkish financial crisis. Levy-Carciente et al. (2015) developed a dynamic network model for banks to analyze the system's sensitivity to external shocks. The authors further test the model using the data for the Venezuelan banking system from 1998 to 2013. Likewise, Poledna et al. (2015) implemented multi-layer network analysis to investigate the Mexican banking system between 2007 and 2013. Khashanah \& Alsulaiman (2016) analyzed the stock market complexity by utilizing the network theory induced by investment behavior.

It is worth mentioning that scholars have implemented various connectivity measures such as reciprocity, interconnectivity, whilst a thorough study introduced a novel methodology known as the population connectedness relied on forecast error variance decompositions of a VAR model (Diebold \& Yllmaz, 2014). The study estimated static and dynamic network volatility interdependence between 13 US financial institutions in 1999:5 and 2010:4. This seminal approach has received overwhelming attention from scholars, and they have computed the network connectedness between financial indicators. Within this category, Diebold et al. (2017) estimated network volatility interdependence among 19 commodity price indices. Along similar lines, Zhang (2017) computed network connectivity between Brent crude oil and six major stock price indices between January 2016 and March 2016 by employing population connectedness. Likewise, Mensi et al. (2018) computed network volatility connectedness among GIPSI stock indices between 2, 2002/1/2, and 2016/5/4. Demirer et al. (2018) computed high-dimensional network connectedness among 150 banks by employing the VAR-LASSO-based population connectedness methodology between 20032014. Caloia et al. (2019) estimated the interconnectedness between five implied volatility indices between August 2008 and December 2017 by implementing the same methodology. Similarly, Kang \& Yoon (2019) computed dynamic network interdependencies among EPUs of G-7 countries, China, and the EU between 1997 and 2016. More recently, Antonakakis et al. (2020) implemented the TVP-VAR-based population connectedness approach and computed network connectedness between monthly four exchange rates' returns.

It should be noted that network structures of connectedness can evolve and be frequencydependent. In this regard, Ellington \& Baruník (2020) developed a frequency-dependent 
network structure that relied on a locally stationary TVP-VAR model using QBLL methods. This seminal approach allows drawing prior shrinkage and estimating uncertainty from the posterior distribution of the network. The study computed short-, medium-, and long-term volatility network connectedness of 496 stocks from the New York Stock Exchange (NYSE) in $2005 / 7 / 5$ and $31,2018 / 8 / 31$.

\section{Empirical Method and Data}

Ellington \& Baruník (2020) construct a dynamic network structure using spectral decomposition of time-varying variance decomposition matrices. The network structure delineates the impact of transitory (short-term frequency) and permanent (long-term frequency) shocks from variable $j$ to the future variance of variable $i$. The model also introduces a dynamic adjacency matrix, which comprises all information indicating the network.

Let $\left(Z_{t, T}\right)_{(1 \leq t \leq T, T \in N)}$ be the $\mathrm{N}$-variate time series with elements $Z_{t, T}=\left(Z_{t, T}^{1}, \ldots, Z_{t, T}^{N}\right)^{T}$. In this setting, $t$ refers the time index, $T$ is an additional index denoting the "sharpness of the local approximation of the time series $\left(Z_{t, T}\right)_{(1 \leq t \leq T, T \in N)}$ by a stationary one" (Ellington \& Baruník, 2020, 7).

Assume that $\left(Z_{t, T}\right)_{(1 \leq t \leq T, T \in N)}$ follows a locally stationary TVP-VAR of lag order of $p$ :

$$
Z_{t, T}=\varphi_{1}(t / T) Z_{t-1, T}+\cdots+\varphi_{p}(t / T) Z_{t-p, T}+\rho_{t, T}
$$

where $\rho_{t, T}=\sum^{(-1 / 2)}(t / T) \gamma_{t, T}$ with $\gamma_{t, T} \sim N I D\left(0, I_{M}\right)$, and time-varying autoregressive coefficients are $\phi(t / T)=\left(\phi_{1}(t / T), \ldots, \phi_{q}(t / T)\right)^{T}$. In a neighborhood of fixed time $\mu_{0}=$ $t_{0} / T$, the process $Z_{t, T}$ is approximated by a stationary process $\tilde{Z}_{t}\left(\mu_{0}\right)$ as:

$$
\left.\tilde{Z}_{t}\left(\mu_{0}\right)=\phi_{1}\left(\mu_{0}\right) \tilde{Z}_{t-1}\left(\mu_{0}\right)+\cdots+\phi_{p}\left(\mu_{0}\right) \tilde{Z}_{(} t-p\right)\left(\mu_{0}\right)+\rho_{t}
$$

with $t \in Z$ and satisfies $\left|Z_{t, T}-\tilde{Z}_{t}\left(t_{0}\right)\right|=O_{q}\left(\left|t / T-\mu_{0}\right|+1 / T\right)$. Thus, the time-varying $\operatorname{VMA}(\infty)$ representation of the process.

$$
Z_{t, T}=\sum_{h=-\infty}^{\infty} \Psi_{t, T}(h) \rho_{t-h}
$$

where $\Psi_{t, T} \approx \phi(t / T, h)$ is a stochastic process with $\sup _{l}\left\|\Psi_{t}-\Psi_{l}\right\|^{2}$ for $1 \leq h \leq t$ as $t \rightarrow \infty$. The spectral density of $Z_{t, T}$ at frequency $w$ is introduced as follows.

$$
S_{Y}(\mu, w)=\sum_{h=-\infty}^{\infty} E\left[\tilde{Z}_{t+h}(\mu) \tilde{Z}_{t}^{T}(\mu)\right] e^{i w h}=\left\{\Psi(\mu) e^{-i w}\right\} \Sigma(\mu)\left\{\Psi(\mu) e^{+i w}\right\}^{T}
$$

Suppose $Z_{t, T}$ is weakly locally stationary process with $\sigma_{k k}^{-1} \sum_{h=0}^{\inf }\left|\left[\Psi(\mu) e^{-i w} \Sigma(\mu)\right]_{j, k}\right|^{2}<$ $\infty, \forall j, k$. "Then, the time-frequency variance decompositions of the $j^{t h}$ variable at a rescaled time $\mu=t_{0} / T$ due to shock in $k^{t h}$ variable on the frequency band $d=(a, b)$ : $a, b \in(-\pi, \pi), a<b$ form a dynamic adjacency matrix" (Ellington \& Baruník, 2020, 8) as:

$$
[\theta(\mu, d)]_{j, k}=\frac{\sigma_{k k}^{-1} \int_{a}^{b}\left|\left[\Psi(\mu) e^{-i w} \Sigma(\mu)\right]_{j, k}\right| d w}{\int_{-\pi}^{\pi}\left[\left\{\Psi(\mu) e^{-i w}\right\} \Sigma(\mu)\left\{\Psi(\mu) e^{-i w}\right\}^{T}\right]_{j, j} d w}
$$


Ellington \& Baruník (2020) introduce local network connectedness as:

$$
C(\mu, d)=100 \times \sum_{\substack{j, k=1 \\ j \neq k}}^{N}[\tilde{\theta}(\mu, d)]_{j, k} / \sum_{\substack{j, k=1 \\ j \neq k}}^{N}[\tilde{\theta}(\mu)]_{j, k}
$$

The local directional connectedness (FROM connectedness) that measures how much of each indicator's $j$ variance due to shocks in other indicators $k \neq j$ is defined as

$$
C_{j \leftarrow .}(\mu, d)=100 \times \sum_{\substack{k=1 \\ k \neq j}}^{N}[\tilde{\theta}(\mu, d)]_{j, k} / \sum_{j, k=1}^{N}[\tilde{\theta}(\mu)]_{j, k}
$$

Likewise, the contribution of $j$ to variances in other indicators is computed as

$$
C_{j \rightarrow \cdot}(\mu, d)=100 \times \sum_{\substack{k=1 \\ k \neq i}}^{N}[\tilde{\theta}(\mu, d)]_{k, j} / \sum_{k, j=1}^{N}[\tilde{\theta}(\mu)]_{k, j}
$$
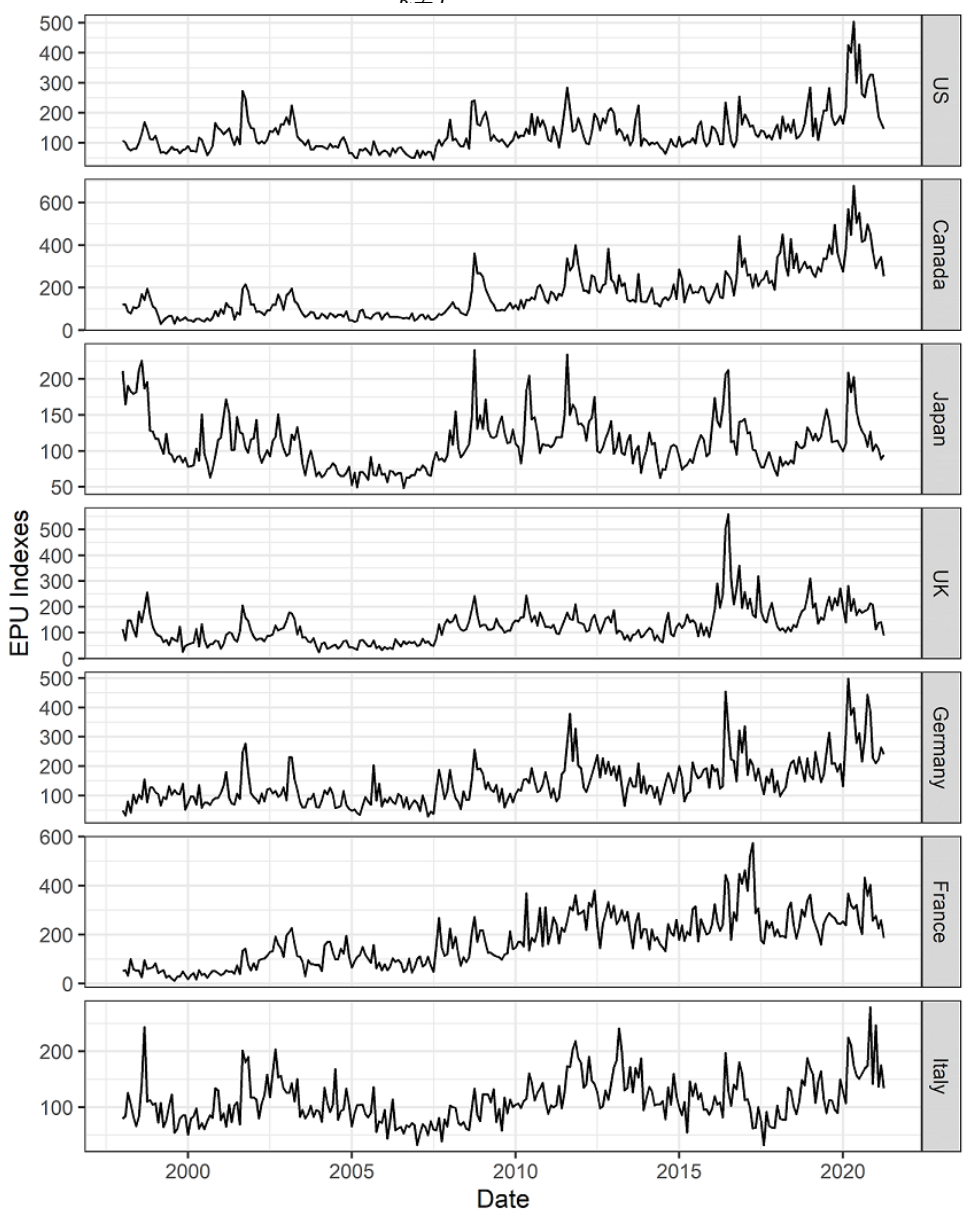

Figure 1: G-7 EPU Indices between January 1998 and April 2021 
In this study, we use monthly EPU data for G-7 countries developed by Baker et al. (2016). The EPU index relies on newspaper coverage frequency. G-7 countries are the world's largest IMF advanced economies, and the GFC emerged in a G-7 state (the US). Besides, the COVID-19 pandemic has sorely hit G-7 countries as well as other developing and advanced economies. This is the main reason for the selection of G-7 countries for the empirical analysis. Our data set spans from 1998:1 to 2021:4 and is gathered from the https://www.policyuncertainty.com/ website.

We depict EPU indices for G-7 countries between January 1998 and April 2021 in Figure 1. The figure delineates that all EPU indices prominently surge during the GFC. EPU indices for EU states (Germany, France, and Italy) create proper signs to the European Sovereign Debt Crisis (ESDC), and accordingly, they significantly climb. Likewise, EPU indices for EU states and the UK soared around June 2016, which coincides with the Brexit referendum. The EPU indices skyrocketed (except for the UK) starting from late 2019 owing to the COVID-19 outbreak.

\section{Results}

On the basis of the observed trends, we compute the network connectedness of G-7 EPU indices by implementing the TVP-VAR based connectedness approach of Ellington \& Baruník (2020). Figure 2 depicts the total dynamic network return connectedness of G-7 EPU indices with median $2.5 \%$ and $97.5 \%$ quantiles. $^{3}$

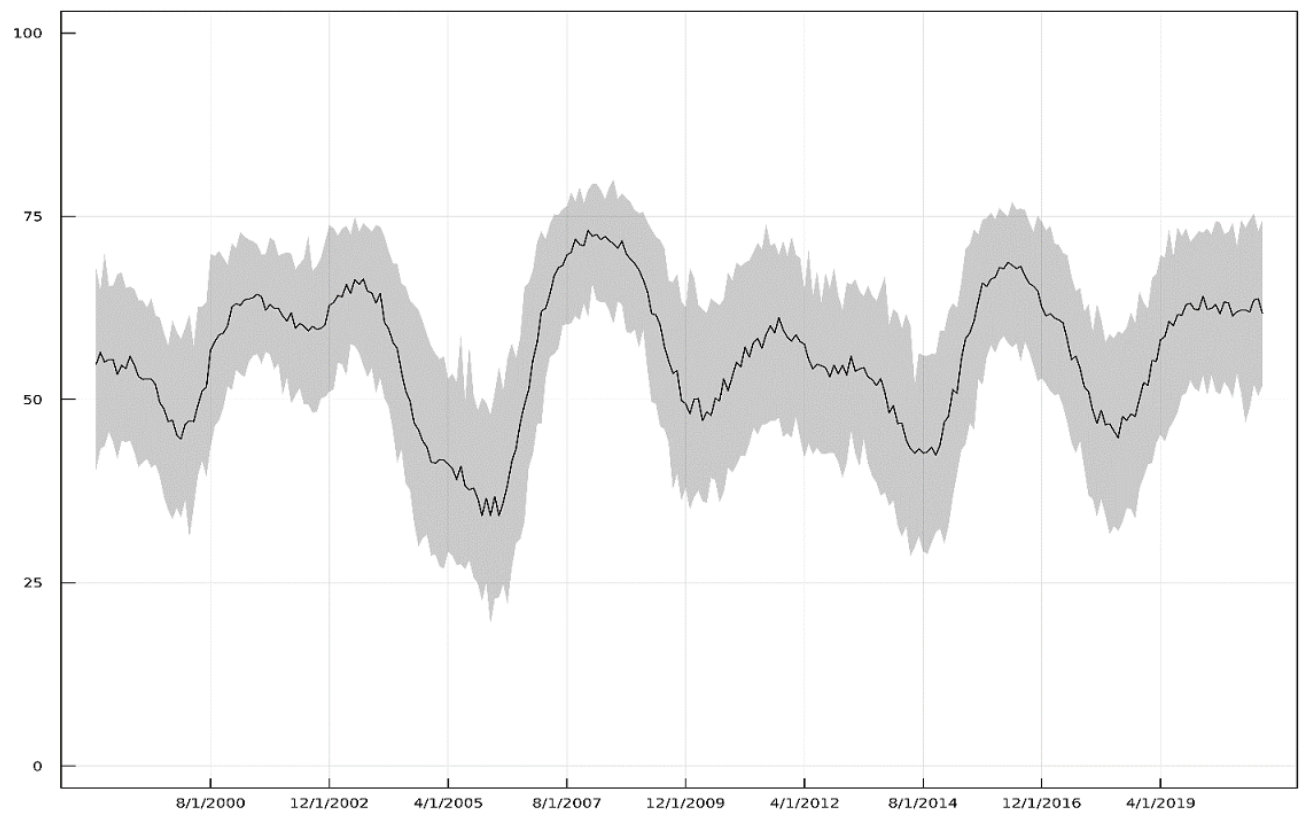

Figure 2: Total Dynamic Network Connectedness of G-7 EPU Indices

\footnotetext{
3 We select the optimal order of the VAR as four by the Akaike Information Criterion (AIC) and the Bayesian Information Criterion (BIC). We run the Julia 1.5.3 program and use libraries available at https:// github.com/barunik/DynamicNets.jl.
} 
The total dynamic network connectedness index of G-7 EPUs swings between 30\% and $75 \%$ over the study period. The index reached its trough in February $2006(34.4 \%)$ and hit its apex in April 2008 (73.0\%), which corresponds to the midst of GFC. The index gradually escalated between May 2010 and October 2011 and November 2014 and April 2016 due to both the ESDC and surges uncertainties owing to the European Immigration Crisis and the Brexit Referendum. ${ }^{4}$ The index steeply plummeted from April 2016 to February 2018, dramatically raised in the June 2018-May 2020 period, and fluctuates thereafter.

In the next step, we estimate posterior medians of short-, medium-, and long-term network connectedness of G-7 EPU indices between May 1998 and April 2021. The estimated posterior medians, as depicted in Figure 3, indicate that the medium-, long-term network connectedness indices exhibit similar patterns, whereas the dynamics of the short-term network connectedness index have distinct characteristics.

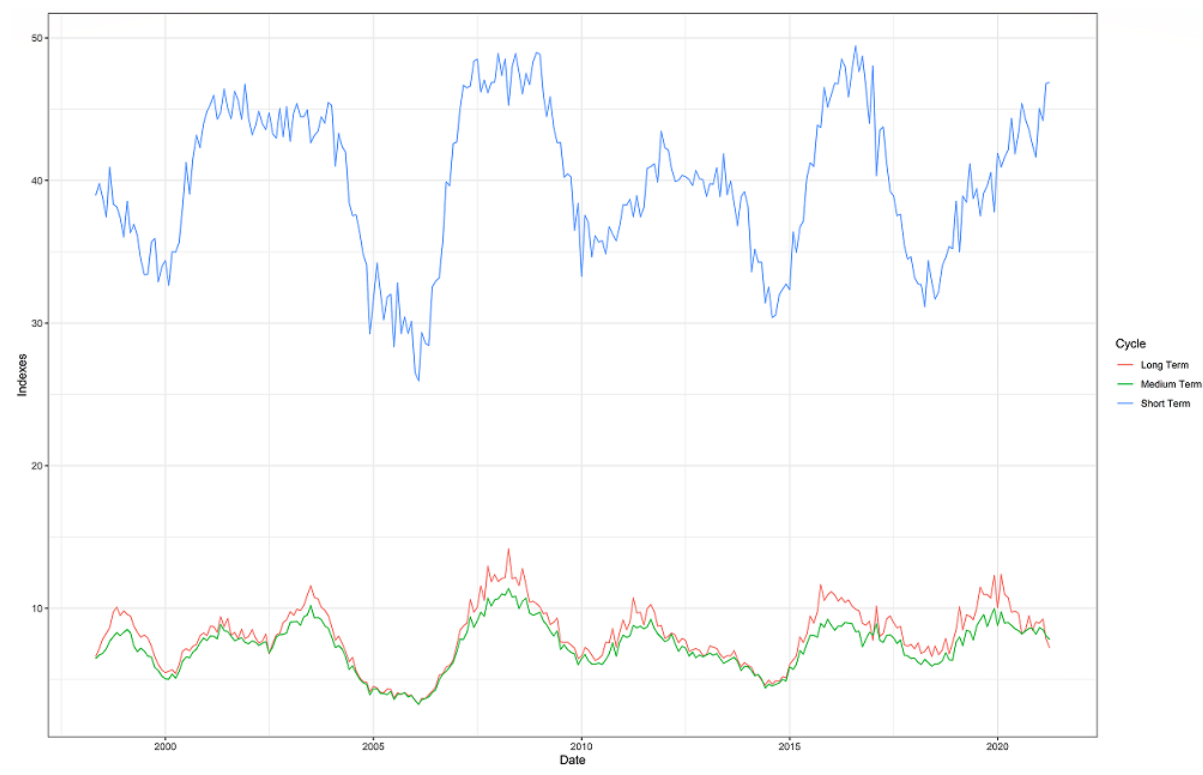

Figure 3: Short-. Medium-, and Long Term Network Connectedness of G-7 EPU Indices

The medium-, and long-term connectedness indices peak in April 2008, while the shortterm connectedness index reached its apex in October 2016. Short-term network connectedness has remarkably surged from $37.8 \%$ in December 2019 to $46.8 \%$ in April 2021, while the medium-, and long-term network connectedness have alleviated. Furthermore, the shortterm network interdependence is stronger than the other two network linkages.

Next, following Ellington \& Baruník (2020), we focus on the short-, and long-term network connectedness topologies reflecting the GFC and the COVID-19 pandemic periods separately. ${ }^{5}$ In network topologies, the size of each node reflects the total TO spillovers from that node, and the thickness of arrows indicates the magnitudes of the spillovers. ${ }^{6}$

${ }^{4}$ See https://www.policyuncertainty.com/media/Global_Annotated_Series.pdf.

${ }^{5}$ We consider the collapse of Lehman Brothers in September 2008 as the start date of the GFC, and the World Health Organization's announcement in March 2020 as the start date of the COVID-19 pandemic.

6 Diebold \& Ylmaz (2014) introduce directional spillovers such that TO directional spillovers reflect spillovers transmitted to that node and FROM spillovers correspond to spillovers received by that node. 

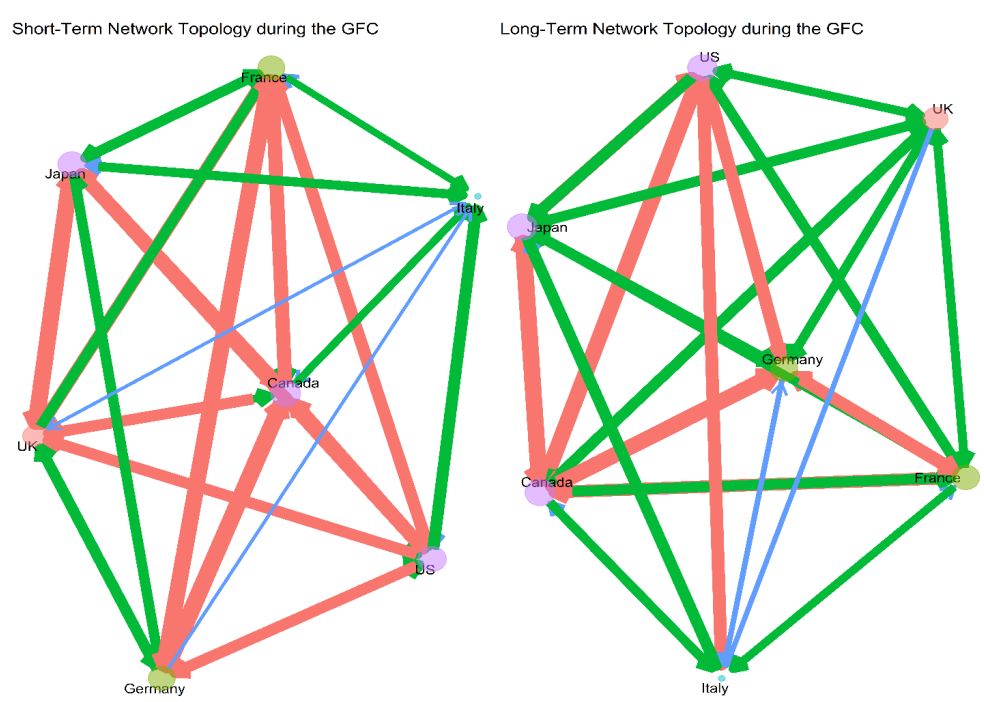

Figure 4: Short- and Long-Term Network Structures of G-7 EPU Connectedness for the GFC

As shown in Figure 4, short-term connectedness is stronger than the long-term connectedness. The short-term connectedness network topology of the GFC indicates that the US catalysts the largest total TO spillovers to other nodes $(58.4 \%)$ and followed by Canada (54.6\%). Since the GFC was originated in the subprime mortgage market of the US and rapidly dispersed to the rest of the world, this finding is consistent with the related literature. Furthermore, our findings provide evidence for the prominent role of geographical proximity in explaining EPU connectedness. On the other hand, Italy transmits the lowest total TO spillovers to other nodes $(23.5 \%)$. The long-term connectedness network topology shows that the US and Canada transmit the largest total TO spillovers with $13.3 \%$ and $13.1 \%$, respectively. Contrariwise, Italy propagates the lowest total TO spillovers (5\%). Prominently plummeting spillovers in the long-term connectedness network topology propose that interdependencies between EPUs are not persistent in the long term.

In the final step, we plot the short-, and long-term EPU connectedness network topologies for the COVID-19 pandemic in Figure 5. Like Figure 4, the short-term connectedness is larger relative to the long-term. The short-term COVID-19 EPU connectedness network indicates that the US (58.6\%) and Germany (55.4\%) transmit the largest total TO spillovers. Meanwhile, the UK propagates the lowest amount of the total TO spillovers (24.9\%). In line with the findings of Al-Thaqeb et al. (2020), the COVID-19 pandemic sorely hit the US economy and led to a dramatic increase in both its EPU and interdependence of the US with other G-7 members. The EPU of Germany dramatically surged from 131.5 in January 2020 to 498.06 in March 2020, which is also accompanied by a prominent increase in its connectedness with other G-7 countries. This may lead to Germany having the secondhighest total TO spillovers among G-7 countries. On the other hand, the EPU of the UK remained relatively silent as compared to other G-7 countries, which may lead the UK to have the lowest total TO spillovers. Likewise, intensified connectedness among EPUs is not persistent in the long term, as clearly pictured in Figure 5. 

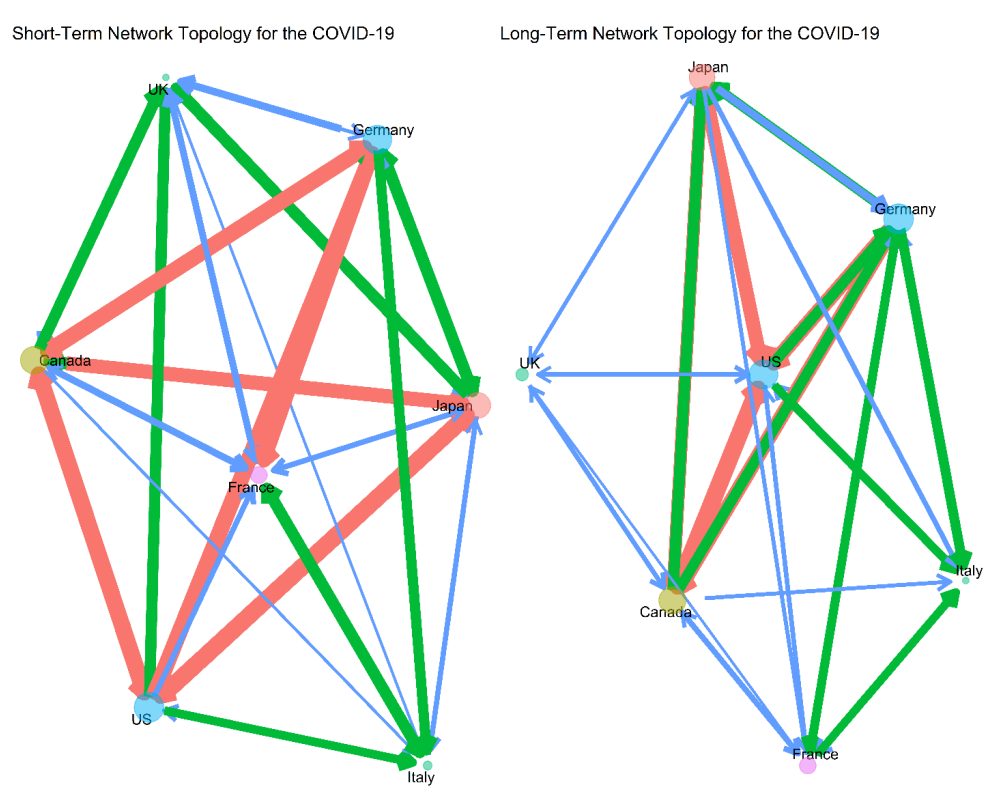

Figure 5: Short- and Long-Term Network Structures of G-7 EPU Connectedness for the COVID-19

\section{Conclusions}

The global economy has witnessed deep and prolonged economic/financial imbalances since the 1929 great depression, yet one of the most acute crises was the GFC. The crisis stemmed from the US financial system and expeditiously spilt throughout the world. In the post-GFC epoch, the world economy has confronted several turbulences, including the ESDC, European Immigration Crisis, the Brexit Referendum, and the US President Election, though the most acute one is arguably the COVID-19 pandemic, which led to a $3.3 \%$ contraction in the 2020 global output (IMF, 2021). Due to the frequency-dependent structure of the network dynamics of connectedness between economic indicators, this study examines G-7 EPU indices connectedness networks on different frequencies. To this end, we employ the seminal methodology of Ellington \& Baruník (2020), which is based on a locally stationary TVP-VAR model using QBLL methods. This methodology allows us to compute short-, medium-, and long-term connectedness among G-7 EPU indexes between January 1998 and April 2021.

The frequency-dependent network structures of G-7 EPU connectedness indicate several results. First, the short-term connectedness of G-7 EPU indices is stronger than the medium-, and long-term interdependence of indices. Second, the medium-, and long-term connectedness indices display similar patterns while the short-term connectedness index is distinct from them. Third, the short-term total dynamic connectedness index hit its vertex in October 2016, though the medium-, and long-term total dynamic connectedness indices reached their maximum levels in April 2008.

Thanks to a precipitous rise in the short-term total dynamic connectedness index during the COVID-19 pandemic, we structure network topologies of G-7 EPU connectedness reflecting the GFC and the COVID-19 epochs. Network analysis indicates the following 
findings. First, the US and Canada catalyst the highest total TO spillovers across G-7 countries during the GFC episode. This result is expected since the GFC was emerged in the US financial system and rapidly dispersed to other countries. Second, the GFC network topology indicates that Italy transmits the lowest total TO spillovers. The US keeps its role of propagating the highest total TO spillovers in the COVID-19 network topology and followed by Germany. On the contrary, the UK transmits the lowest total TO spillovers.

This study has an important policy suggestion. Since interconnectedness among G-7 EPUs dramatically amplifies around financial/geopolitical bursts due to the strong interdependencies among countries, authorities and policy-makers should closely monitor contagion among both emerging and advanced economies' economic policy uncertainties. To this end, modern measurement techniques and risk management strategies can be helpful.

\section{References}

Abel, A. B., \& Eberly, J. C. (1996). Optimal Investment with Costly Reversibility. The Review of Economic Studies, 63(4), 581-593. doi:10.2307/2297794

Al-Thaqeb, S. A., Algharabali, B. G., \& Alabdulghafour, K. T. (2020). The Pandemic and Economic Policy Uncertainty. International Journal of Finance \&3 Economics, 1-11. doi:10.1002/ijfe.2298

Antonakakis, N., Chatziantoniou, I., \& Gabauer, D. (2020). Refined Measures of Dynamic Connectedness based on Time-Varying Parameter Vector Autoregressions. Journal of Risk and Financial Management, 13(4). doi:10.3390/jrfm13040084

Bagheri, E., \& Ebrahimi, S. B. (2020). Estimating Network Connectedness of Financial Markets and Commodities. Journal of Systems Science and Systems Engineering, 29, 572-589. doi:10.1007/s11518-020-5465-1

Baker, S. R., Bloom, N., \& Davis, S. J. (2016). Measuring economic policy uncertainty. The Quarterly Journal of Economics, 131(4), 1593-1636. doi:10.1093/qje/qjw024

Balcılar, M., Demirer, R., Gupta, R., \& van Eyden, R. (2017). The impact of US policy uncertainty on the monetary effectiveness in the Euro area. Journal of Policy Modeling, 39(6), 1052-1064. doi:10.1016/j.jpolmod.2017.09.002

Balcılar, M., Gabauer, D., \& Umar, Z. (2021). Crude Oil futures contracts and commodity markets: New evidence from a TVP-VAR extended joint connectedness approach. Resources Policy, 73, 102219. doi:10.1016/j.resourpol.2021.102219

Barrett, P., Das, S., Magistretti, G., Pugacheva, E., \& Wingender, P. (2021). After-Effects of the COVID-19 Pandemic: Prospects for Medium-Term Economic Damage (IMF Working Paper No. WP/21/203). International Monetary Fund. https://www.imf.org/en/Publications/WP/Issues/2021/07/30/After-Effects-of -the-COVID-19-Pandemic-Prospects-for-Medium-Term-Economic-Damage-462898. 
Beaman, L., BenYishay, A., Magruder, J., \& Mobarak, A. M. (2021, June). Can Network Theory-Based Targeting Increase Technology Adoption? American Economic Review, 111 (6), 1918-43. doi:10.1257/aer.20200295

Bernanke, B. S. (1983). Irreversibility, Uncertainty, and Cyclical Investment. The Quarterly Journal of Economics, 98(1), 85-106. doi:10.2307/1885568

Billio, M., Getmansky, M., Lo, A. W., \& Pelizzon, L. (2012). Econometric measures of connectedness and systemic risk in the finance and insurance sectors. Journal of Financial Economics, 104 (3), 535-559. doi:10.1016/j.jfineco.2011.12.010

Byrne, J. P., \& Davis, E. P. (2004). Permanent and temporary inflation uncertainty and investment in the United States. Economics Letters, 85(2), 271-277. doi:10.1016/j.econlet.2004.04.015

Caloia, F. G., Cipollini, A., \& Muzzioli, S. (2019). How do normalization schemes affect net spillovers? a replication of the diebold and yilmaz (2012) study. Energy Economics, 84, 104536. doi:10.1016/j.eneco.2019.104536

Cimini, R. (2015). Eurozone network "Connectedness" after fiscal year 2008. Finance Research Letters, 14, 160-166. doi:10.1016/j.frl.2015.05.003

Cresswell, K. M., Worth, A., \& Sheikh, A. (2010). Actor-Network Theory and its role in understanding the implementation of information technology developments in healthcare. BMC Medical Informatics and Decision Making, 10(67), 1-11. doi:10.1186/1472-6947$10-67$

De Pooter, M., Favara, G., Modugno, M., \& Wu, J. (2021). Monetary policy uncertainty and monetary policy surprises. Journal of International Money and Finance, 112, 102323. doi:10.1016/j.jimonfin.2020.102323

Demirer, M., Diebold, F. X., Liu, L., \& Yilmaz, K. (2018). Estimating global bank network connectedness. Journal of Applied Econometrics, 33(1), 1-15. doi:10.1002/jae.2585

Diebold, F. X., Liu, L., \& Yilmaz, K. (2017). Commodity Connectedness (Working Paper No. 23685). National Bureau of Economic Research. doi:10.3386/w23685

Diebold, F. X., \& Yllmaz, K. (2014). On the network topology of variance decompositions: Measuring the connectedness of financial firms. Journal of Econometrics, 182(1), 119134. doi:10.1016/j.jeconom.2014.04.012

Ellington, M., \& Baruník, J. (2020). Dynamic Networks in Large Financial and Economic Systems. Available at SSRN 3651134. doi:10.2139/ssrn.3651134

Fenwick, T. J. (2010). (un)Doing standards in education with actor-network theory. Journal of Education Policy, 25(2), 117-133. doi:10.1080/02680930903314277

Fernández-Villaverde, J., Guerrón-Quintana, P., Kuester, K., \& Rubio-Ramírez, J. (2015). Fiscal Volatility Shocks and Economic Activity. American Economic Review, 105(11), 3352-3384. doi:10.1257/aer.20121236 
Geng, J.-B., Chen, F.-R., Ji, Q., \& Liu, B.-Y. (2021). Network connectedness between natural gas markets, uncertainty and stock markets. Energy Economics, 95, 105001. doi:10.1016/j.eneco.2020.105001

Gong, X.-L., Liu, X.-H., Xiong, X., \& Zhang, W. (2019). Financial systemic risk measurement based on causal network connectedness analysis. International Review of Economics E3 Finance, 64, 290-307. doi:10.1016/j.iref.2019.07.004

Hassett, K. A., \& Metcalf, G. E. (1999). Investment with Uncertain Tax Policy: Does Random Tax Policy Discourage Investment. The Economic Journal, 109(457), 372-393. doi:10.1111/1468-0297.00453

IMF. (2021). World Economic Outlook. https://www.imf.org/en/Publications/WEO/ Issues/2021/03/23/world-economic-outlook-april-2021.

Iyamu, T., \& Mgudlwa, S. (2018). Transformation of healthcare big data through the lens of actor network theory. International Journal of Healthcare Management, 11(3), 182-192. doi:10.1080/20479700.2017.1397340

Kang, S. H., \& Lee, J. W. (2019). The network connectedness of volatility spillovers across global futures markets. Physica A: Statistical Mechanics and its Applications, 526, 120756. doi:10.1016/j.physa.2019.03.121

Kang, S. H., \& Yoon, S.-M. (2019). Dynamic connectedness network in economic policy uncertainties. Applied Economics Letters, 26(1), 74-78. doi:10.1080/13504851.2018.1438580

Khashanah, K., \& Alsulaiman, T. (2016). Network theory and behavioral finance in a heterogeneous market environment. Complexity, 21 (S2), 530-554. doi:10.1002/cplx.21834

Kuzubaş, T. U., Ömercikoğlu, I., \& Saltoğlu, B. (2014). Network centrality measures and systemic risk: An application to the Turkish financial crisis. Physica A: Statistical Mechanics and its Applications, 405, 203-215. doi:10.1016/j.physa.2014.03.006

Levy-Carciente, S., Kenett, D. Y., Avakian, A., Stanley, H. E., \& Havlin, S. (2015). Dynamical macroprudential stress testing using network theory. Journal of Banking E Finance, 59, 164-181. doi:10.1016/j.jbankfin.2015.05.008

Mensi, W., Al Rababa'a, A. R., Vo, X. V., \& Kang, S. H. (2021). Asymmetric spillover and network connectedness between crude oil, gold, and Chinese sector stock markets. Energy Economics, 98, 105262. doi:10.1016/j.eneco.2021.105262

Mensi, W., Boubaker, F. Z., Al-Yahyaee, K. H., \& Kang, S. H. (2018). Dynamic volatility spillovers and connectedness between global, regional, and GIPSI stock markets. Finance Research Letters, 25, 230-238. doi:10.1016/j.frl.2017.10.032

Murdoch, J. (2001). Ecologising Sociology: Actor-Network Theory, Coconstruction and the Problem of Human Exemptionalism. Sociology, 35(1), 111-133. doi:10.1017/S0038038501000074 
Mützel, S. (2009). Networks as Culturally Constituted Processes: A Comparison of Relational Sociology and Actor-network Theory. Current Sociology, 57(6), 871-887. doi:10.1177/0011392109342223

Poledna, S., Molina-Borboa, J. L., Martínez-Jaramillo, S., van der Leij, M., \& Thurner, S. (2015). The multi-layer network nature of systemic risk and its implications for the costs of financial crises. Journal of Financial Stability, 20, 70-81. doi:10.1016/j.jfs.2015.08.001

Reboredo, J. C., Ugolini, A., \& Aiube, F. A. L. (2020). Network connectedness of green bonds and asset classes. Energy Economics, 86, 104629. doi:10.1016/j.eneco.2019.104629

Rodrik, D. (1991). Policy uncertainty and private investment in developing countries. Journal of Development Economics, 36(2), 229-242. doi:10.1016/0304-3878(91)90034$\mathrm{S}$

Romer, P. M. (1990). Endogenous Technological Change. Journal of Political Economy, 98(5, Part 2), S71-S102. doi:10.1086/261725

Shim, Y., \& Shin, D.-H. (2016). Analyzing China's Fintech Industry from the Perspective of Actor-Network Theory. Telecommunications Policy, 40(2), 168-181. doi:10.1016/j.telpol.2015.11.005

Singh, V. K., Nishant, S., \& Kumar, P. (2018). Dynamic and directional network connectedness of crude oil and currencies: Evidence from implied volatility. Energy Economics, 76, 48-63. doi:10.1016/j.eneco.2018.09.018

Valente, T. W., \& Pitts, S. R. (2017). An Appraisal of Social Network Theory and Analysis as Applied to Public Health: Challenges and Opportunities. Annual Review of Public Health, 38(1), 103-118. doi:10.1146/annurev-publhealth-031816-044528

Weiske, C., Petzold, K., \& Schad, H. (2015). Multi-Local Living - The Approaches of Rational Choice Theory, Sociology of Everyday Life and Actor-Network Theory. Tijdschrift voor Economische en Sociale Geografie, 106(4), 392-408. doi:10.1111/tesg.12157

Zhang, D. (2017). Oil shocks and stock markets revisited: Measuring connectedness from a global perspective. Energy Economics, 62, 323-333. doi:10.1016/j.eneco.2017.01.009

Zhang, D., \& Broadstock, D. C. (2020). Global financial crisis and rising connectedness in the international commodity markets. International Review of Financial Analysis, 68, 101239. doi:10.1016/j.irfa.2018.08.003

Zhang, Z., \& Heydon, R. (2016). The changing landscape of literacy curriculum in a Sino-Canada transnational education programme: an actor-network theory informed case study. Journal of Curriculum Studies, 48(4), 547-564. doi:10.1080/00220272.2015.1090626 